\title{
Pattern of Dermatologic Disorders in Infants
}

\author{
HUSSAIN M $^{\mathrm{a}}$, YASMEEN BHN ${ }^{\mathrm{b}}$, ISLAM SMS $^{\mathrm{c}}$, AMIN N $^{\mathrm{d}}$, BEGUM J $^{\mathrm{e}}$
}

\begin{abstract}
Background: Pattern of skin disorders varies in different ages and in different countries. This pattern also varies among children of different ages. The prevalence pattern reflects socioeconomic, environmental and hygienic condition of a community.

Objective: This study was planned to evaluate the magnitude of skin diseases and pattern of various dermatoses among children below one year of age in a major tertiary care hospital in the capital of Bangladesh.

Method: A retrospective study was undertaken to determine the pattern of skin diseases during the period of January 2010 to June 2012 at the dermatology outpatient department of a tertiary care pediatric hospital in the capital of Bangladesh. Data were analyzed to determine the pattern of skin diseases in children less than one year of age, seen at the Out Patient Department (OPD) of Dhaka Shishu Hospital (DSH).
\end{abstract}

\section{Introduction}

Skin comprises about $15 \%$ of the total body weight ${ }^{1}$. Infants have a higher surface area-to-volume ratio in comparison to older children \& adults. There are important structural and physiological differences between skin of adult and that of infant, the function of skin remaining essentially the same at all phases of life, including: barrier, photo-protection, thermoregulation, immune surveillance, vitamin synthesis, prevention of insensible fluid loss, and sensory perception. ${ }^{2}$ Infant skin is found to have higher water content and is able to absorb more water and lose excess water faster than adult skin. Micro structural difference relates to thinner stratum corneum and papillary dermis in infant skin. ${ }^{3}$ There is greatly increased mortality of premature infants due to microbial invasion with impaired barrier function. ${ }^{4}$ The stratum corneum of infant is $10-15$ micron thick.

a. Dr. Mahfuza Hussain, Consultant Dermatologist, Dhaka Shishu Hospital, Sher-e- Banglanagar, Dhaka.

b. Dr. BH Nazma Yasmeen, Associate Professor \& Head of the department of Pediatric, Northern Medical College \& Hospital, Dhaka.

c. Dr. SM Shaheedul Islam, Associate Professor, Ibrahim Cardiac Hospital \& Research Institute, Dhaka.

d. Dr. Nurul Amin, Research Specialist, Ibrahim Cardiac \& Research Institute, Dhaka.

e. Dr. Jamila Begum, Sr Consultant, Gynae and Obstetrics Department, LAB AID Specialized Hospital, Dhaka

Correspondence: Dr Mahfuza Hussain, Consultant Dermatologist, Dhaka Shishu Hospital, Sher-e- Banglanagar, Dhaka, Email:mafihaq@gmail.com, Tel:+8801711535590

Received: 02 December, 2012

Accepted: 09 July, 2013
Results: A total of 36608 cases were seen at the dermatological OPD of DSH out of which 9293 (25.39\%) were less than one year of age. The frequency of distribution of dermatological diseases was more in male patients $58.2 \%$ than in female patients 41.8\%. Neonates were 649 (7\%) and rest were infants were 8644 (93\%). Eczema was the most common skin disorder (55.41\%) followed by impetigo \& other bacterial infections (27.55\%) and scabies (23.44\%). Cutaneous fungal infections were $13.99 \%$, miliaria was $4.9 \%$, erythema neonatorum $4.42 \%$, urticaria was $2.18 \%$, bullous lesions $1.45 \%$, haemangioma $1.17 \%$ and pityriasis alba $1.15 \%$. About 16 other dermatological disorders were indentified each of which was less than $1 \%$ in distribution.

Conclusions: Eczema, superficial bacterial infections, scabies and superficial fungal infections were most common dermatological problem in infants.

Key words: Skin disease, Infant

(Birdem Med J 2013; 3(2): 80-84)

Damage to it increases skin permeability, and may increase potential for skin or systemic infection, or systemic toxicity to topical chemicals or medication. Direct barrier injury can occur because of increased fragility of infant skin even from removal of adhesive tapes and monitors and thus increasing local permeability. Given the estimated $50 \%$ incidence of atopic dermatitis in infants, there are other reasons for barrier function to be impaired at baseline. ${ }^{5}$ In intrauterine life the skin is protected by vernix caseosa and the surrounding amniotic fluid. After birth the parameters of skin like thickness, skin $\mathrm{pH}$, stratum corneum maturation continuously get adapted to extra-uterine environment unlike adult skin which remains in a steady state $^{6}$. "Smooth as a baby's bottom" summarizes the popular notion of infant skin; however there are significant structural and functional differences that make infant skin more susceptible to certain problems. During the first years of life, there are considerable developments of the skin and subcutaneous fat that warrant handling infants differently. ${ }^{7}$ The age related changes in the dermis can be observed histologically and biochemically. Collagen synthesis decreases and collagen fiber thickens with age and elastic fibers steadily increase in numbers and thickness. ${ }^{8}$

Many if not all dermatologic conditions in children exhibit different patterns compared to adults. ${ }^{9}$ Children form a major group in our population and pediatric dermatological cases are seen by both dermatologist and pediatricians in clinical practice. ${ }^{10}$ The close liaison between the pediatrician and the dermatologist will help in early prevention and 
TB are the highest, where MDR-TB makes 20 percent of all new cases.

As the tuberculosis chemotherapy era evolved, increasing cases of drug resistance continued to occur mainly as a result of inadequate regimens and nonadherence to therapy. Researchers initially suspected that these resistant organisms had reduced fitness and thus could be classified as being less virulent. This assumption was reversed in the 1990s with the rise in MDR tuberculosis. Substantial attention was focused upon New York City (NY, USA) where a virulent and transmissible strain had spread among immunocompromised populations. Awareness of tuberculosis drug resistance was refocused with a study presented in August, 2006, at the XVI International AIDS Conference in Toronto, Canada, which described an epidemic of XDR tuberculosis in a rural hospital in KwaZulu-Natal Province, South Africa.

To combat this situation a more focused and comprehensive strategy is needed. In Bangladesh the national tuberculosis control programme (NTP) introduced DOTS (Directly observed treatment short clourse) strategy in 1993 and gradually expanded. By mid 1998 it covered all upazillas. In 2002 DOTS started in Dhaka Metropolitan City and by 2006 the geographical Coverage was $100 \%$. In 2006; The STOP TB Strategy with special emphasis on NSN(new smear negative), EP(extra pulmonary), Child TB, MDR-TB and TB/HIV was taken. NTP objectives are detection of cases $>70 \%$ (Achieved in 2006), treat successfully $>85 \%$ (Achieved in 2003), reach MDG related targets of halving TB mortality and prevalence by 2015; So far NTP has established National TB Reference Lab (NTRL) at NIDCH, Dhaka.Regional TB Reference Lab (RTRL) at Rajshahi, Chittagong .One more is to be established soon in Khulna.

Number of Enrolled MDR-TB patients is1098 (up to December 2012). Treatment success rate up to 2009 cohort $=64 \%$

Country's 1st nationwide drug resistance survey conducted shows MDR 1.4\% \& $28.5 \%$ among new and previously treated TB cases respectively.

\section{References:}

1. Fact sheet: World TB day, 2011; Bangladesh tuberculosis Control Programme. (www.stoptb.org/..infactsheets/ fast.facts.asp.) 